2. G. Birkhoff and S. MacLane, A survey of modern algebra, MacMillan, 1953.

3. P. R. Halmos, Algebraic Logic, I-Monadic Boolean algebras, Compositia Math. vol. 12 (1955) pp. 217-249.

4. J. C. C. McKinsey and Alfred Tarski, The algebra of topology, Ann. of Math. vol. 45 (1944) pp. 141-191.

University of Chicago

\title{
ON HARMONIC MAPPINGS ${ }^{1}$
}

JOHANNES C. C. NITSCHE

1. Suppose that the functions $x=x(\alpha, \beta), y=y(\alpha, \beta)$ define a oneto-one harmonic mapping of the unit disc $\Gamma$ in the $\alpha, \beta$-plane $(\alpha+i \beta$ $=\gamma$ ) onto a convex domain $C$ in the $x, y$-plane $(x+i y=z)$. The origin is assumed to be fixed. Introducing two functions $F(\gamma)$ and $G(\gamma)$ which, in $\Gamma$, depend analytically upon the variable $\gamma$ we may write $z=\operatorname{Re} F(\gamma)+i \operatorname{Re} G(\gamma)$. The purpose of the present paper is (i) to give a new proof of a lemma which, in a special form, was first used by T. Radó [13] and which was proved in general by L. Bers (see [2, Lemma 3.3]), ${ }^{2}$ (ii) to derive an improved value for an important constant first introduced by $\mathrm{E}$. Heinz [3]. The proofs will be very simple due to the fact that there is a close connection between univalent harmonic mappings and the minimal surface equation (see e.g. $[11$, footnote 2]) and also the differential equation

$$
\phi_{x x} \phi_{y y}-\stackrel{2}{\phi_{x y}}=1 .
$$

The connection with the latter equation was exploited by K. Joergens [8] for the study of the solutions of (1). One can, however, proceed one step further by introducing a mapping which was invented by $\mathrm{H}$. Lewy $[10]$ for Monge-Ampère equations.

2. Let $z=\operatorname{Re} F(\gamma)+i \operatorname{Re} G(\gamma)$ be a harmonic mapping with the properties mentioned above. Then the expression

Presented to the Society August 20, 1956 and November 17, 1956; received by the editors September 10, 1957.

1 This paper was prepared under Contract $\mathrm{N}$ onr-710(16) between the University of Minnesota and the Office of Naval Research.

${ }^{2}$ It has been shown by H. Hopf (cf. [7, p. 133 and 5, pp. 91-92]) that the combination of Heinz's inequality with Schwarz's lemma yields a sharper result. 


$$
\phi=\frac{1}{2} \operatorname{Im}\left(F \bar{G}+\int_{0}^{\gamma}\left(F G^{\prime}-F^{\prime} G\right) d \gamma\right)
$$

may be regarded as a function $\phi(x, y)$ of $x$ and $y$, defined in $C$ (see $\mathrm{K}$. Joergens $[8, \mathrm{p} .339])$. By a straightforward computation it can be verified that $\phi(x, y)$ is a solution of the Equation (1). In fact, one obtains

$$
\begin{gathered}
p=-\operatorname{Im} G(\gamma), \quad q=\operatorname{Im} F(\gamma), \\
r=\left|G^{\prime}\right|^{2} \cdot\left[\operatorname{Im} F^{\prime} \bar{G}^{\prime}\right]^{-1}, \quad s=-\left[\operatorname{Re} F^{\prime} \bar{G}^{\prime}\right] \cdot\left[\operatorname{Im} F^{\prime} \bar{G}^{\prime}\right]^{-1}, \\
t=\left|F^{\prime}\right|^{2} \cdot\left[\operatorname{Im} F^{\prime} \bar{G}^{\prime}\right]^{-1} .
\end{gathered}
$$

Here $p, q, r, s, t$ are abbreviations for $\phi_{x}, \phi_{y}, \phi_{x x}, \phi_{x y}, \phi_{y y}$, as usual. According to a lemma of H. Lewy [9], $\operatorname{Im}\left(F^{\prime} \bar{G}^{\prime}\right)=x_{\alpha} y_{\beta}-x_{\beta} y_{\alpha} \neq 0$ in $\Gamma$. It may be assumed that $\operatorname{Im}\left(F^{\prime} \bar{G}^{\prime}\right)>0$. Then $\phi_{x x}>0$. Now consider, in $C$, the functions

$$
u=u(x, y)=x+p(x, y), \quad v=v(x, y)=y+q(x, y)
$$

and put $u+i v=w$. For any two points $z_{1}$ and $z_{2}$ in $C$ the following inequality holds true

$$
\begin{array}{r}
\left(x_{2}-x_{1}\right)\left[p\left(x_{2}, y_{2}\right)-p\left(x_{1}, y_{1}\right)\right]+\left(y_{2}-y_{1}\right)\left[q\left(x_{2}, y_{2}\right)-q\left(x_{1}, y_{1}\right)\right] \\
=\tilde{r}\left(x_{2}-x_{1}\right)^{2}+2 \tilde{s}\left(x_{2}-x_{1}\right)\left(y_{2}-y_{1}\right)+\tilde{t}\left(y_{2}-y_{1}\right)^{2} \geqq 0 .
\end{array}
$$

Here $\tilde{r}, \tilde{s}, \tilde{t}$ stand for the values of $r, s, t$ in a point of the segment connecting $z_{1}$ with $z_{2}$. Substitute (4) into (5):

(6) $\left(x_{2}-x_{1}\right)^{2}+\left(y_{2}-y_{1}\right)^{2} \leqq\left(x_{2}-x_{1}\right)\left(u_{2}-u_{1}\right)+\left(y_{2}-y_{1}\right)\left(v_{2}-v_{1}\right)$ and hence

$$
\left|z_{2}-z_{1}\right| \leqq\left|w_{2}-w_{1}\right|
$$

equality holding only if $z_{1}=z_{2}$ (see $H$. Lewy [10]). Therefore the mapping (4) is one-to-one and it enlarges distances. Denote by $\Omega$ the image domain of $C$ under this mapping. On the other hand, going back to the definitions of $x, y$ and $p, q$ one finds

$$
w=F(\gamma)+i G(\gamma) \equiv W(\gamma) .
$$

That is to say the domain $\Omega$ is also the schlicht conformal image of $\Gamma$ under the mapping function $W(\gamma)$.

3. It is easy to see that, starting out with a solution $\phi(x, y)$ of (1), the inverse mapping $w \rightarrow z$ under all circumstances is harmonic. Furthermore it turns out that the expression $f=2 \bar{z}-\bar{w}$ which can be regarded as a function of $u$ and $v$ is an analytic function of $w$. The 
inequality $|d f / d w|<1$ which is satisfied by its derivative has interesting consequences, see [12].

4. The lemma in question states that there cannot exist a schlicht harmonic mapping of the unit disc $\Gamma$ onto the whole $z$-plane. The proof is obvious since, if $C$ would be the whole $z$-plane then $\Omega$ would have to be the whole $w$-plane. But, at the same time, $\Omega$ is the conformal image of $\Gamma$. This is not possible.

5. Suppose now that $C$, like $\Gamma$, is the unit disc $|z|<1$. E. Heinz [3] has established an inequality

$$
\left.x_{\alpha}^{2}+x_{\beta}^{2}+y_{\alpha}^{2}+y_{\beta}^{2}\right]_{\gamma=0} \geqq \mu .
$$

Here his constant $\mu$ is independent of the individual harmonic mapping under consideration. Heinz found $\mu \geqq 2-(8 / \pi) \sum_{n=2}^{\infty} n^{-2}=0.358$. Using the relations derived above one obtains the formula

$$
x_{\alpha}^{2}+x_{\beta}^{2}+y_{\alpha}^{2}+y_{\beta}^{2}=\frac{r+t}{2+r+t} \cdot\left|\frac{d W}{d \gamma}\right|^{2} .
$$

Remembering the properties of the mapping (4) we know that $\Omega$ contains at least a circle of radius 1 . Hence, by Schwarz's lemma, $|d W(0) / d \gamma| \geqq 1$. In fact, the sign of equality cannot hold since $\partial(u, v) / \partial(x, y)=2+r+t \geqq 4$. Furthermore $1 / 2 \leqq(r+t) /(2+r+t)<1$. Combining these two inequalities we conclude

$$
\mu \geqq 1 / 2 \text {. }
$$

6. We wish to mention that $\mathrm{H}$. $\mathrm{Hopf}^{3}$ has given another simple proof of the value $1 / 2$ for the constant $\mu$. A similar inequality to (9) holds also for more general univalent mappings, see P. Berg [1], E. Heinz $[4 ; 5]$. However, remaining with the harmonic mappings: the best value of $\mu$ is not known. ${ }^{4}$ If one takes the polynomial solution $\phi(x, y)=c x^{2} / 2+y^{2} / 2 c$ then $\Omega$ is an ellipse with the semiaxes $1+c$ and $1+1 / c$. A computation yields

$$
\lim _{c \rightarrow \infty} \frac{r+t}{2+r+t}=1, \quad \lim _{c \rightarrow \infty}\left|\frac{d W(0)}{d \gamma}\right|=\frac{4}{\pi},
$$

and hence

\footnotetext{
${ }^{3}$ In a letter of October 26, 1956.

${ }^{4}$ Added in proof: A refinement of the preceding method yields even $\mu \geqq 0.64$, as will be shown elsewhere. Therefore, referring to Richert's example for an upper bound, one knows: $0.64 \leqq \mu \leqq 27 / 2 \pi^{2}$.
} 


$$
\lim _{c \rightarrow \infty}\left[x_{\alpha}^{2}+x_{\beta}^{2}+y_{\alpha}^{2}+y_{\beta}^{2}\right]_{\gamma=0}=16 / \pi^{2}
$$

By an example of H. E. Richert (cf. E. Hopf [6, p. 802]) it is, however, known that the value $16 / \pi^{2}$ is too large

\section{LITERATURE}

1. P. Berg, On univalent mappings by solutions of linear elliptic partial differential equations, Trans. Amer. Math. Soc. vol. 84 (1957) pp. 310-318.

2. L. Bers, Isolated singularities of minimal surfaces, Ann. of Math. vol. 53 (1951) pp. 364-386.

3. E. Heinz, Ueber die Loesungen der Minimalflaechengleichung, Nachr. Akad. Wiss. Goettingen, Math.-Phys. K1. (1952) pp. 51-56.

4. E. Heinz, Ueber gewisse elliptische Systeme von Differentialgleichungen zweiter Ordnung mit Anwendung auf die Monge-Ampère'sche Gleichung, Math. Ann. vol. 131 (1956) pp. 411-428.

5. E. Heinz, On certain non-linear elliptic differential equations and univalent mappings, Technical Report No. 63, Stanford University, 1957.

6. E. Hopf, On an inequality for minimal surfaces $z=z(x, y)$, Journal of Rational Mechanics and Analysis vol. 3 (1953) pp. 519-522 and 801-802.

7. K. Joergens, Ueber die Loesungen der Differentialgleichung $r t-s^{2}=1$, Math. Ann. vol. 127 (1954) pp. 130-134.

8. - Harmonische Abbildungen und die Differentialgleichung $r t-s^{2}=1$, Math. Ann. vol. 129 (1955) pp. 330-344.

9. H. Lewy, On the nonvanishing of the Jacobian in certain one-to-one mappings, Bull. Amer. Math. Soc. vol. 42 (1936) pp. 689-692.

10. - A priori limitations for solutions of Monge-Ampère equations II, Trans. Amer. Math. Soc. vol. 41 (1937) pp. 365-374.

11. J. Nitsche, Ueber eine mit der Minimalflaechengleichung zusammenhaengende analytische Funktion und den Bernsteinschen Satz, Archiv der Mathematik vol. 7 (1956) pp. 417-419.

12. - Elementary proof of Bernstein's theorem on minimal surfaces, Ann. of Math. vol. 66 (1957) pp. 543-544.

13. T. Rad6, Zu einem Satze von Bernstein ueber Minimalflaechen im Grossen, Math. Ztschr. vol. 26 (1927) pp. 559-565.

UNIVERSITY OF MinNESOTA 